\title{
Collective behavior of Franck-Condon excited states and energy transfer in double helices
}

\section{Dimitra Markovitsi, Delphine Onidas, Thomas Gustavsson, Francis Talbot and Elodie Lazzarotto}

\author{
Laboratoire Francis Perrin, CEA/DSM/DRECAM/SPAM - CNRS URA 2453, CEA Saclay, \\ 91191 Gif-sur-Yvette, France
}

\section{Materials}

Nucleotides, dAMP and TMP, were obtained from Sigma Aldrich and dissolved in water. Polymers, poly(dA).poly(dT) (ca. 2000 bp), poly(dAdT).poly(dAdT) (200 - 400 bp) and poly(dA), were purchased from Amersham Biosciences and were dissolved in phosphate buffer $(\mathrm{pH}=6.8 ; 0.1 \mathrm{M}$ $\left.\mathrm{NaH}_{2} \mathrm{PO}_{4}, 0.1 \mathrm{M} \mathrm{Na}_{2} \mathrm{HPO}_{4}, 0.25 \mathrm{M} \mathrm{NaCl}\right)$. The melting temperature of poly(dA).poly(dT) and poly(dAdT).poly(dAdT), determined by recording the optical density at $260 \mathrm{~nm}$, was $85^{\circ} \mathrm{C}$ and $74^{\circ} \mathrm{C}$, respectively. Temperature control was achieved by means of a LAUDA ECOLINE 003.

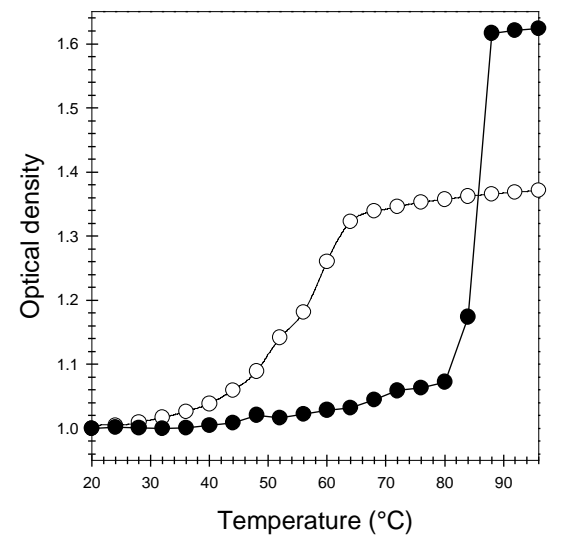

Fig. S1. Melting curve of poly(dA).poly(dT) (black circles). For comparison, the curve corresponding to the oligomer $(d A)_{20 .}(d T)_{20}$ (Eurogentec) is also shown (white circles).

\section{Experimental details}

Steady-state absorption and fluorescence spectra were recorded with a Perkin Lamda 900 and a SPEX Fluorolog-2 spectrofluorometer, respectively, according to the procedure described elsewhere. ${ }^{1}$ Fluorescence quantum yields $\left(\phi_{x}\right)$ were determined using quinine sulfate dihydrate in $0.1 \mathrm{M} \mathrm{HCl}\left(\phi_{s}=\right.$ $0.59){ }^{2}$

The time-resolved measurements used as excitation source the third harmonic of a mode-locked Ti-sapphire laser $(267 \mathrm{~nm})$. The repetition rate was $76 \mathrm{MHz}$ for upconversion measurements but, for time-correlated single photon counting operation, it was reduced to $236 \mathrm{KHz}$ by means of a pulsepicker (Coherent Model 9200). 
The fluorescence upconversion set-up is described in detail elsewhere. ${ }^{3}$ Temporal scans were made in both parallel ( $\left.\mathrm{I}_{\mathrm{par}}\right)$ and perpendicular $\left(\mathrm{I}_{\mathrm{perp}}\right)$ mode by controlling the polarization of the exciting beam with a half-wave plate. The total fluorescence decays $F(t)$ were calculated according to: $F(t)=I_{p a r}(t)+$ $2 \mathrm{I}_{\text {per }}(\mathrm{t})$. The full width at half maximum of the Gaussian apparatus function, determined by separate measurements of the water Raman line at $295 \mathrm{~nm}$, was about $400 \mathrm{fs}$. The time-resolution of our set-up is judged to be $100 \mathrm{fs}$ after de-convolution.

The time-correlated single photon counting set-up used a Becker \& Hickl GmbH PC card. A cutoff filter Schott WG 295 was placed in front of a SPEX monochromator. The detector was a microchannel plate (R1564 U Hamamatsu) providing an instrumental response function of $60 \mathrm{ps}$ (fwhm). Parallel and perpendicular components were determined by means of a Glan Thomson prism. The $\mathrm{G}$ factor $(0.88 \pm 0.02)$ was determined by the tail-matching method using protonated guanosine $(\mathrm{pH}$ $=2.5)$. The fluorescence anisotropy was calculated as $\mathrm{r}(\mathrm{t})=\left(\mathrm{I}_{\mathrm{par}}(\mathrm{t})-\mathrm{GI}_{\mathrm{per}}(\mathrm{t})\right) /\left(\mathrm{I}_{\mathrm{par}}(\mathrm{t})+2 \mathrm{GI}_{\text {per }}(\mathrm{t})\right)$. The zero time was taken at the half maximum of the signal rise. Specific caution had to be taken in order to avoid contamination of the signals by emission from photoproducts. This is understandable if we consider that, for example, the (6-4) thymine dimer has a fluorescence quantum yield $\left(c a .10^{-2}\right)^{4}$ which is two orders of magnitude higher than that of the DNA duplexes $\left(c a .10^{-4}\right)$. In the upconversion measurements, about $20 \mathrm{ml}$ of solutions were kept flowing through a $0.4 \mathrm{~mm}$ quartz cell (optical density at $267 \mathrm{~nm}$ : $c a .0 .2$ ); the pulse intensity at the surface of the cell was $200 \mathrm{MW} / \mathrm{cm}^{2}$. In the single photon counting measurements, $0.6 \mathrm{ml}$ of solutions were contained in a $10 \times 2 \mathrm{~mm}^{2}$ quartz cell (optical densities at $267 \mathrm{~nm}: c a$. 1) which was displaced perpendicularly to the exciting beam $(0.7 \mathrm{~cm} / \mathrm{sec})$; the laser was defocused at the sample surface leading to a pulse intensity of $10 \mathrm{KW} / \mathrm{cm}^{2}$. Under such conditions, successive measurements yielded identical signals. All measurements were carried out at $20^{\circ} \mathrm{C}$.

\section{Steady-state fluorescence spectra}

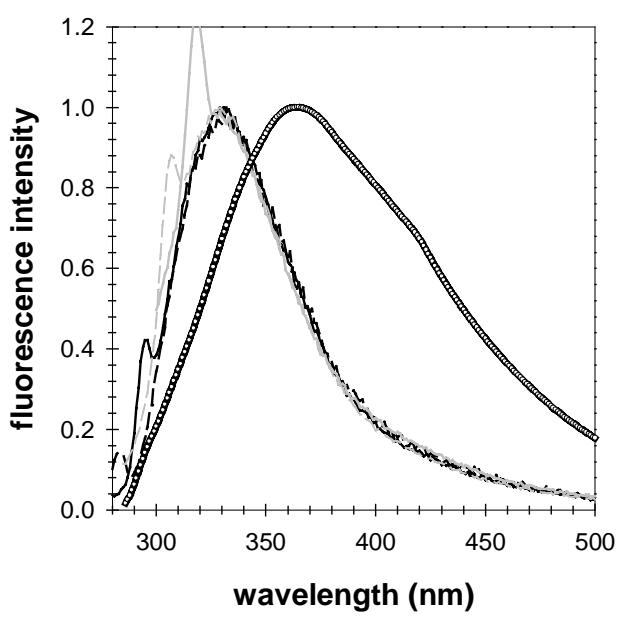

Fig. S2. Steady-state fluorescence spectra of poly $(d A)$.poly $(d T)$ recorded for excitation wavelengths 255, 265, 275 and $285 \mathrm{~nm}$ (overlapping thin lines). The sharp peaks correspond to the Raman line of water. For comparison, the spectrum of poly( $d A$ ) obtained for $\lambda$ ex $=265 \mathrm{~nm}$ is also shown (circles). 


\section{Fit of the fluorescence decays}

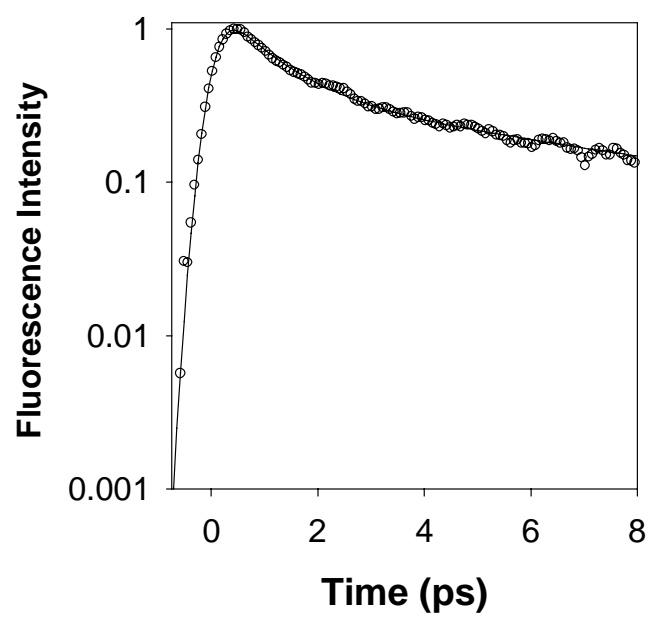

Fig. S3.Example of a fit of a fluorescence decay obtained for poly $(d A)$.poly $(d T)$ by upconversion at $420 \mathrm{~nm}$. The trace is fitted with the bi-exponential function $0.72 \exp (-t / 0.94)+10 \exp (-t / 8.3)$, convoluted with a Gaussian apparatus function defined by a full width at half maximum of $400 \mathrm{fs}$. Only the first picoseconds of the total decay are shown in order to illustrate the lack of any rise time.

\section{REFERENCES}

(1) Onidas, D.; Markovitsi, D.; Marguet, S.; Sharonov, A.; Gustavsson, T. J. Phys. Chem. B 2002, 106, 11367- 11374.

(2) Velapoldi, R. A.; Mielenz, K. D. A fluorescence standard reference material: quinine sulfate dihydrate; U. S. Government Printing Office, Washington, D. C.: Washington, 1980. (3) Gustavsson, T.; Sharonov, A.; Markovitsi, D. Chem. Phys. Lett. 2002, 351, 195-200; Gustavsson, T.; Sharonov, A.; Onidas, D.; Markovitsi, D. Chem. Phys. Lett. 2002, 356, 4954.

(4) Blais, J.; Douki, T.; Vigny, P.; Cadet, J. Photochem. Photobiol. 1994, 59, 402-404. 